\title{
Lower Prevalence of Osteoporosis in Patients with COPD Taking Anti-Inflammatory Compounds for the Treatment of Diabetes: Results from COSYCONET
}

Kathrin Kahnert,' Rudolf A Jörres, (D) ${ }^{2}$ Tanja Lucke, (iD) ${ }^{2}$

Franziska C Trudzinski, (iD) ${ }^{3}$

Pontus Mertsch,' Christiane Bickert,' Joachim H Ficker, (D) ${ }^{4}$ Jürgen Behr,'

Robert Bals, ${ }^{5}$ Henrik Watz, ${ }^{6}$

Tobias Welte, (iD) 7 Claus F Vogelmeier, ${ }^{8}$ Peter Alter (iD) 8

On behalf of COSYCONET Study Group

'Department of Medicine V, University Hospital, LMU Munich, Comprehensive Pneumology Center Munich (CPC-M), Member of the German Center for Lung Research (DZL), Munich, Germany; ${ }^{2}$ Institute and Outpatient Clinic for Occupational, Social and Environmental Medicine, University Hospital, LMU Munich, Comprehensive Pneumology Center Munich (CPC-M), Member of the German Center for Lung Research (DZL), Munich, Germany; ${ }^{3}$ Department of Pneumology and Critical Care Medicine, Thoraxklinik, University of Heidelberg, Translational Lung Research Center Heidelberg (TLRC-H), Member of the German Center for Lung Research (DZL), Heidelberg, Germany; ${ }^{4}$ Department of Respiratory Medicine, Nürnberg General Hospital, Paracelsus Medical University, Nürnberg, Germany; ${ }^{5}$ Department of Internal Medicine V - Pulmonology, Allergology, Intensive Care Medicine, Saarland University Hospital, Homburg, Germany; ${ }^{6}$ Pulmonary Research Institute at LungenClinic Grosshansdorf, Airway Research Center North (ARCN), Member of the German Center for Lung Research (DZL), Grosshansdorf, Germany;

${ }^{7}$ Clinic for Pneumology, Hannover Medical School, Member of the German Center for Lung Research (DZL), Hannover, Germany; ${ }^{8}$ Department of Medicine, Pulmonary and Critical Care Medicine, University of Marburg (UMR), Germany, Member of the German Center for Lung Research (DZL), Marburg, Germany

Correspondence: Kathrin Kahnert Department of Medicine V, University Hospital, LMU Munich, Comprehensive Pneumology Center Munich (CPC-M), Member of the German Center for Lung Research (DZL),

Munich, Germany

Tel +49894400 52590

Fax +4989440054905

Email kathrin.kahnert@med.uni-muenchen.de
Background: Patients with chronic obstructive pulmonary disease (COPD) often have osteoporosis and diabetes as comorbid conditions. Anti-diabetic medication, including metformin, has protective effects on osteoporosis in experimental studies. We therefore studied whether patients with COPD receiving anti-diabetic medication had a lower osteoporosis prevalence in a large COPD cohort, COSYCONET.

Methods: Assessment of osteoporosis was based on patients' reports of physician-based diagnoses and the presence of disease-specific medication. The predictive value of physical characteristics, lung function, comorbidities, cardiovascular medication, and the use of antiinflammatory diabetes medication, including metformin, sulfonylureas, glinides or DPP4I, was evaluated using logistic regression analysis. ClinicalTrials.gov: NCT01245933.

Results: In total, 2222 patients were eligible for analysis (863 [39\%] female, mean age 65 y), 515 of whom had higher symptoms and exacerbations (Global Initiative for Chronic Obstructive Lung Disease group D). Osteoporosis was present in $15.8 \%$ of the overall cohort, and in $24.1 \%$ of GOLD D patients. Regression analyses identified the following as associated with osteoporosis $(\mathrm{p}<0.05)$ : female sex, higher age, lower body-mass index, asthma, higher air trapping, oral steroids, and cardiovascular medication. Although oral anti-diabetic medication was overall not associated with a lower prevalence of osteoporosis $(\mathrm{p}=0.131)$, anti-inflammatory anti-diabetic medication $(\mathrm{p}=0.009)$ and metformincontaining therapy $(\mathrm{p}=0.039)$ were. This was driven by GOLD D patients.

Conclusion: In a large COPD cohort, anti-inflammatory diabetes therapy, including metformin, was associated with a lower prevalence of osteoporosis, especially in patients with higher symptoms and exacerbations. These findings suggest a protective effect of common antidiabetic medication on osteoporosis, possibly as a result of attenuated systemic inflammation.

Keywords: chronic obstructive pulmonary disease, oral corticosteroids, inhaled corticosteroids, anti-inflammatory, metformin, diabetes

\section{Introduction}

Osteoporosis is a major comorbidity of chronic obstructive pulmonary disease (COPD). ${ }^{1-7}$ In addition to generic osteoporosis risk factors, such as female sex, higher age, smoking, cachexia and reduced physical capacity, a range of COPD-related risk factors are known to also increase osteoporosis risk, including the severity of pulmonary impairment, vitamin D deficiency, or systemic inflammation, ${ }^{8,9}$ with systemic 
inflammation also an independent predictor of low bone mineral density (BMD) in patients with stable COPD. ${ }^{10}$ Diabetes mellitus is also a common comorbidity in COPD, ${ }^{11}$ and there is evidence that inflammatory pathways are involved in the pathogenesis of this disease. ${ }^{12}$ Besides decreased BMD, more frequently observed in type 1 diabetes, decreased bone quality is associated with increased fragility, especially in type 2 diabetes. ${ }^{13}$ This suggests that in patients with comorbid COPD and diabetes, systemic inflammation related to both diseases may contribute to the development of osteoporosis. Conversely, anti-inflammatory medication with systemic action, in particular anti-diabetic medication containing metformin, sulfonylureas, glinides or DPP4I, ${ }^{11,14,15}$ could have the potential to reduce the risk of osteoporosis. $^{13,16}$

A further contributing factor in COPD may be the fact that many patients are treated with inhaled corticosteroids (ICS), ${ }^{17}$ with a significant proportion receiving oral corticosteroids (OCS). ${ }^{18}$ OCS use clearly promotes the development and progression of osteoporosis, ${ }^{19}$ whereas findings for ICS use are equivocal in patients with COPD - although there is an increased risk of osteoporosis in patients with asthma, especially when receiving high-dose ICS. $^{20}$

Recent in-vitro and animal studies have evaluated the role of metformin in bone turnover independent of the presence of diabetes. $^{21-24}$ Anti-diabetic drugs, especially metformin, have the potential to prevent corticosteroid-induced bone loss, including via mechanisms to suppress bone resorption and stimulate bone formation. ${ }^{25}$ Taken together, this suggests that patients with COPD could be appropriate to examine potential effects of anti-diabetic medication on the development of osteoporosis. This could be particularly true for patients with a high disease burden ${ }^{18}$ as is the case for those classified by the Global Initiative for Chronic Obstructive Lung Disease (GOLD) as "group D", ${ }^{26}$ who would therefore be expected to more frequently use ICS and/or OCS therapy. Based on this, data from the German COPD and Systemic Consequences Comorbidities Network (COSYCONET) cohort were analyzed to evaluate whether the use of anti-diabetic medication is associated with a lower adjusted prevalence of osteoporosis. $^{4}$

\section{Materials and Methods Study Population}

The present study used baseline data (visit 1) from the COSYCONET COPD cohort, a multi-center study investigating the role of comorbidities in patients with COPD
(ClinicalTrials.gov: NCT01245933). COSYCONET was conducted in accordance with the Declaration of Helsinki, has been approved by the ethics committees of all study centers, and all patients gave their written informed consent. Detailed information on the recruitment process, inclusion/ exclusion criteria and assessments can be found elsewhere. ${ }^{4}$

\section{Assessments}

All measurements were performed when patients had stable disease, defined as the absence of moderate or severe exacerbations within the last four weeks. ${ }^{4}$ Postbronchodilator ${ }^{4}$ lung function data included forced expiratory volume in $1 \mathrm{~s}\left(\mathrm{FEV}_{1}\right)$ and forced vital capacity (FVC), each as percent predicted, ${ }^{27}$ and the ratio of residual volume to total lung capacity (RV/TLC), as a measure of air trapping/hyperinflation. Patients were classified as GOLD grades $1-4$ based on $\mathrm{FEV}_{1}$ categories, ${ }^{26}$ and GOLD groups A-D on symptoms and exacerbation history according to GOLD 2017 criteria, ${ }^{26}$ using the modified Medical Research Council dyspnea scale (mMRC). Smoking was categorized as current smoking versus former or never-smoking. These analyses only included patients of GOLD grades $1-4,{ }^{26,28}$ who had complete data for age, sex, body-mass index (BMI), smoking status, FEV1, FVC, RV, TLC and GOLD grouping.

\section{Comorbidities and Medication}

Patients were asked to bring all their medication to the study visit. $^{29}$ The presence of comorbidities, including osteoporosis and diabetes, was assessed in a structured interview from patient reports of physician-based diagnoses $^{4}$ and supplemented by disease-specific medication. ${ }^{29}$ Coronary artery disease, cardiac failure and myocardial infarction were analyzed individually and were also summarized into a combined "cardiac disease" variable. Cardiovascular medication included beta-blockers, renin-angiotensin-aldosterone system inhibitors, angiotensin-receptor antagonists, angiotensin II receptor blockers, calcium-channel blockers and diuretics. Anti-diabetes medication comprised metformin, sulfonylureas, glinides, dipeptidyl peptidase 4 inhibitors (DPP4I), incretin mimetics and insulin. Medication containing metformin, sulfonylureas, glinides or DPP4I was considered antiinflammatory. ${ }^{14,15}$ We also evaluated the category of metformin-containing medication, ${ }^{25}$ alone or in combination with other anti-diabetes drugs, as well as that of oral antidiabetes drugs in general. 


\section{Data Analysis}

Groups with and without osteoporosis were compared using either unpaired $t$-tests or chi-square statistics as appropriate. Binary logistic regression analysis was employed to assess the dependence of osteoporosis on risk factors. For this purpose, sets of variables were successively added, until the final set of predictors was reached. This process started with the set of demographic data, then lung function, then comorbidities, then medication. We retained all variables that were significant $(\mathrm{p}<$ 0.05 , two-sided) and also any non-significant variables that were considered relevant confounders from a clinical perspective. The analyses were performed for the total data set and also for single GOLD groups or combinations of groups. To reveal whether groups of COPD patients with different disease severity showed a different association with diabetes medication, we performed analyses stratified according to GOLD groups. This approach was chosen, as the introduction of an indicator variable for these categories implicitly assumes a linear effect, while it may be that the degree of association is affected not the mean level. To properly account for this would have needed the introduction of several interaction terms, thereby complicating the analysis. All analyses were performed using SPSS (Version 27.0.0.0, Armonk, NY, US). We additionally checked the results of the regression analyses by propensity score matching, using the method of full matching, as done previously for the effects of respiratory medication on the heart. ${ }^{30}$

\section{Results}

\section{Baseline Characteristics}

The study population comprised 2222 patients, 351 (15.8\%) of whom had a diagnosis of osteoporosis, with osteoporosis prevalence across GOLD groups $\mathrm{A} / \mathrm{B} / \mathrm{C} / \mathrm{D}$ of $11.1 / 16.0 / 14.5 / 24.1 \%$. Further characteristics stratified by presence or absence of osteoporosis are given in Table 1. There were significant differences between the two groups

Table I Patient Characteristics Including Demographics, Lung Function, COPD Categories, Major Comorbidities and Medication

\begin{tabular}{|c|c|c|c|c|}
\hline \multirow[t]{2}{*}{ Variable } & All & No Osteoporosis & Osteoporosis & \multirow[t]{2}{*}{ p value } \\
\hline & $\mathbf{N}=2222$ & $N=187 \mid$ & $\mathbf{N}=35 \mathrm{I}$ & \\
\hline \multicolumn{5}{|l|}{ Demographics } \\
\hline Age (y) & $65.0 \pm 8.4$ & $64.8 \pm 8.6$ & $66.2 \pm 7.5$ & 0.005 \\
\hline Sex $m / f$ & 1359 (6I.2\%)/863 (38.8\%) & $1225(65.5 \%) / 646$ (34.5\%) & $134(38.2 \%) / 217$ (6I.8\%) & $<0.001$ \\
\hline BMI $\left(\mathrm{kg} / \mathrm{m}^{2}\right)$ & $26.6 \pm 5.2$ & $26.9 \pm 5.2$ & $25.2 \pm 4.9$ & $<0.001$ \\
\hline Smoking status (active) & $552(24.8 \%)$ & $48 \mid(25.7 \%)$ & 7I (20.2\%) & 0.029 \\
\hline \multicolumn{5}{|l|}{ Lung function } \\
\hline FEV (\% predicted) & $52.8 \pm 18.4$ & $53.4 \pm 18.4$ & $49.5 \pm 18.4$ & $<0.001$ \\
\hline FVC (\% predicted) & $78.5 \pm 18.9$ & $79.2 \pm 19.0$ & $75.1 \pm 18.2$ & $<0.001$ \\
\hline RV/TLC (\%) & $54.4 \pm 11.0$ & $53.6 \pm 10.9$ & $58.4 \pm 10.6$ & $<0.001$ \\
\hline GOLD grades I/2/3/4 & $200 / 945 / 852 / 225$ & $|74 / 82| / 702 / 174$ & $26 /|24 /| 50 / 5 \mid$ & 0.001 \\
\hline GOLD groups $A / B / C / D$ & $861 / 556 / 290 / 515$ & $765 / 467 / 248 / 391$ & $96 / 89 / 42 / 124$ & $<0.001$ \\
\hline \multicolumn{5}{|l|}{ Comorbidities } \\
\hline Asthma & 425 (19.1\%) & 325 (I7.4\%) & $100(28.5 \%)$ & $<0.001$ \\
\hline Diabetes & $288(13.0 \%)$ & $253(13.5 \%)$ & $35(10.0 \%)$ & 0.069 \\
\hline Arterial hypertension & 1254 (56.4\%) & $1042(55.7 \%)$ & $212(60.4 \%)$ & 0.103 \\
\hline Cardiac disease & $46 \mathrm{I}(20.7 \%)$ & $383(20.5 \%)$ & 78 (22.2\%) & 0.458 \\
\hline \multicolumn{5}{|l|}{ Medication } \\
\hline ICS & 1460 (65.7\%) & 1200 (64.1\%) & $260(74.1 \%)$ & $<0.001$ \\
\hline OCS & $280(12.6 \%)$ & $200(10.7 \%)$ & $80(22.8 \%)$ & $<0.001$ \\
\hline Cardiac medication & I 327 (59.7\%) & 1087 (58.1\%) & $240(68.4 \%)$ & $<0.001$ \\
\hline Oral anti-diabetes medication & $222(10.0 \%)$ & $199(10.6 \%)$ & $23(6.6 \%)$ & 0.019 \\
\hline All anti-inflammatory diabetes medication & 175 (7.9\%) & $162(8.7 \%)$ & $13(3.7 \%)$ & 0.002 \\
\hline Any metformin-containing medication & $140(6.3 \%)$ & $|3|$ (7.0\%) & $9(2.6 \%)$ & 0.002 \\
\hline
\end{tabular}

Note: Data are mean \pm standard deviation or number (percent).

Abbreviations: $\mathrm{FEV}_{1}$, forced expiratory volume in I s; FVC, forced vital capacity; RV/TLC, ratio of residual volume to total lung capacity determined by body plethysmography; BMI, body mass index; ICS, inhaled corticosteroids; OCS, oral corticosteroids. 
for sex, age, BMI, smoking status, GOLD grades and groups, $\mathrm{FEV}_{1}, \mathrm{FVC}, \mathrm{RV} / \mathrm{TLC}$, and the presence of asthma (all $\mathrm{p}<0.05$ ), but not the presence of diabetes or cardiac disease (either as a summary score or the single items; not shown). As we performed a separate analysis for patients of GOLD group D, baseline characteristics were compared between group D patients and the pooled groups $\mathrm{ABC}$ (Supplemental Table 1).

\section{Medication}

Of 351 patients with osteoporosis, 105 (29.9\%) received osteoporosis-specific medication; in 15 (4.3\%) patients the osteoporosis diagnosis was based solely on the presence of this disease-specific medication. ICS use was reported overall by $1460(65.7 \%)$ patients, with use higher in those with osteoporosis compared to those without $(74.1 \%$ versus $64.1 \%, \mathrm{p}<0.001$, Table 1$)$. OCS therapy was used by 280 $(12.6 \%)$ patients, again with use higher in the osteoporosis group (22.8\% versus $10.7 \%, \mathrm{p}<0.001)$, overlapping with ICS use. Overall, 1327 patients (59.7\%) were receiving any type of cardiac medication (including diuretics), with yet again use higher in those with osteoporosis $(68.4 \%$ versus $58.1 \%, \mathrm{p}<0.001)$. Oral anti-diabetic medication was being used by $222(77.1 \%)$ of the 288 patients with diabetes: antiinflammatory diabetes medication (defined as above) in 175 patients $(60.8 \%)$, and specifically metformin-containing therapy in 140 patients $(48.6 \%)$. The percentages of patients receiving anti-inflammatory $(3.7 \%$ versus $8.7 \%, p=0.002)$ or specifically metformin-containing therapy $(2.6 \%$ versus $7.0 \%, \mathrm{p}=0.002$ ) were lower in the osteoporosis group. Comparisons between GOLD group D and groups ABC are shown in Supplemental Table 1, illustrating the higher percentage of ICS, OCS and cardiac medication use by patients in group D.

\section{Associations Between Osteoporosis and Predictors \\ Analysis of the Total Study Cohort}

The process of variable selection is shown in Table 2 . Using the demographic data as predictors, osteoporosis

Table 2 Results of Logistic Regression Analyses During Several Steps, Leading to Final Step 8

\begin{tabular}{|c|c|c|c|c|c|c|c|c|}
\hline Predictor & Step I & Step 2 & Step 3 & Step 4 & Step 5 & Step 6 & Step 7 & Step 8 \\
\hline $\begin{array}{l}\text { Demographics } \\
\text { Age (per } 10 \text { y) } \\
\text { Sex (female vs male) } \\
\text { BMI }\left(\mathrm{kg} / \mathrm{m}^{2} \text { ) }\right. \\
\text { Smoking status (active) }\end{array}$ & $\begin{array}{l}1.307 * * * \\
3.087 * * * \\
0.942 * * * \\
0.699 *\end{array}$ & & $\begin{array}{l}1.252^{* *} \\
2.797 * * * \\
0.951 * * * \\
0.729 *\end{array}$ & $\begin{array}{l}1.247 * * \\
3.178 * * * \\
0.931 * * * \\
0.733^{*}\end{array}$ & $\begin{array}{l}1.215^{*} \\
2.77 I^{* * *} \\
0.946 * * * \\
0.756^{\mathrm{a}}\end{array}$ & $\begin{array}{l}1.215^{*} \\
2.848 * * * \\
0.945 * * * \\
0.810\end{array}$ & $\begin{array}{l}1.179 * \\
2.827 * * * \\
0.94 I^{* * *} \\
0.841\end{array}$ & $\begin{array}{l}1.182 * \\
2.829 * * * \\
0.942 * * * \\
0.850\end{array}$ \\
\hline $\begin{array}{l}\text { Lung function } \\
\text { FEV } \text { (\% predicted) } \\
\text { FVC (\% predicted) } \\
\text { RV/TLC (\%) }\end{array}$ & & $\begin{array}{l}1.007 \\
1.009 \\
1.063^{* * *}\end{array}$ & $1.028^{* * *}$ & & $1.029 * * *$ & $1.023 * * *$ & $1.022 * * *$ & $1.022^{* * *}$ \\
\hline $\begin{array}{l}\text { Comorbidities } \\
\text { Asthma } \\
\text { Sleep apnea } \\
\text { Diabetes } \\
\text { Arterial hypertension } \\
\text { Cardiac disease } \\
\text { Hyperuricemia } \\
\text { Hyperlipidemia }\end{array}$ & & & & $\begin{array}{l}1.650^{* * *} \\
1.157 \\
0.870^{\mathrm{a}} \\
1.342^{*} \\
1.363^{\mathrm{a}} \\
1.202 \\
1.073\end{array}$ & $\begin{array}{l}1.746 * * * \\
0.857 \\
1.319 * \\
1.359 *\end{array}$ & $\begin{array}{l}1.576 * * \\
0.837 \\
1.278 \\
1.383 *\end{array}$ & $\begin{array}{l}1.620 * * * \\
0.828 \\
0.902 \\
\quad 1.257^{\mathrm{a}}\end{array}$ & $\begin{array}{l}1.643 * * * \\
1.371 \\
0.912 \\
1.259\end{array}$ \\
\hline $\begin{array}{l}\text { Medication } \\
\text { ICS } \\
\text { OCS } \\
\text { Cardiac medication } \\
\text { Diabetes medication }\end{array}$ & & & & & & $\begin{array}{r}1.222^{\mathrm{a}} \\
1.956^{* * * *}\end{array}$ & $\begin{array}{l}\mathrm{I} .198 \\
\mathrm{I} .863^{* * *} \\
\mathrm{I} .742^{* *}\end{array}$ & $\begin{array}{l}\mathrm{I} .199 \\
1.818^{* * *} \\
1.723^{* *} \\
0.358^{* *}\end{array}$ \\
\hline
\end{tabular}

Notes: Odds ratios (Exp [B]) values are shown. Predictors revealed as statistically significant were kept in the subsequent steps of analyses. Significance is denoted as follows: ${ }^{*}<<0.05,{ }^{*} \mathrm{p}<0.01,{ }^{*} *{ }^{*}<0.00 \mathrm{I}{ }^{a}{ }^{2}$ These predictors were included in the following steps, even when not being statistically significant, as they were rated as of potential clinical relevance. In the final analysis we used either any oral anti-diabetes medication, or any anti-inflammatory anti-diabetes medication, or any metformin-containing medication (see Results); the table shows the results for any anti-inflammatory anti-diabetes medication (see Table 2 for details).

Abbreviations: BMI, body mass index; ICS, inhaled corticosteroids; OCS, oral corticosteroids. 
was associated with older age, female sex, lower BMI and active smoking status. Using lung function parameters as predictors, it was linked only to higher RV/TLC. When combining the two sets of variables, a higher RV/TLC, older age, female sex, lower BMI and active smoking status remained significant. Regarding comorbidities as predictors (Table 2), only asthma and hypertension were significantly linked to osteoporosis, with a tendency for cardiac disease. When further combining comorbidities with both demographic characteristics and RV/TLC, older age, female sex, higher BMI, asthma, hypertension and higher RV/TLC remained significant. We thus kept asthma and hypertension in the further analyses as robust predictors but also smoking status, diabetes and cardiac disease despite being not significant to account for their presence when analyzing the effects of diabetes and cardiac medication.

In the next step, ICS and OCS use were added as predictors. Only OCS use was significantly related to osteoporosis. Despite this, we kept ICS in the further analyses since they might be relevant in subgroups. Cardiovascular medication was introduced as the next predictor; it was significantly associated with an increased prevalence of osteoporosis. In the final step, we added anti-diabetic medication as predictor (Table 2). There was no significant relationship between osteoporosis and the presence of any oral anti-diabetic medication $(\mathrm{p}=$ 0.131). However, anti-inflammatory anti-diabetic medication $(\mathrm{p}=0.009)$ was associated with a reduction in the prevalence of osteoporosis $(\mathrm{p}=0.008)$, as was metformincontaining medication $(\mathrm{p}=0.039)$ (Table 3 and Figure 1). As can be seen in Table 3, the detrimental effect of cardiac medication remained significant.

\section{Analysis Stratified According to GOLD Groups}

The associations with anti-inflammatory diabetes medication and with metformin found in the total population of patients were significant only in GOLD group D $(p=0.006)$, and not in groups $\mathrm{A}, \mathrm{B}$ or $\mathrm{C}$. We therefore pooled these groups in the analysis. In this pooled group, the associations were still not

Table 3 Results of Logistic Regression Analyses in Either the Total Population of Patients or Those in GOLD Group D, Demonstrating the Significant Effect of Anti-Inflammatory Diabetes Medication in Both

\begin{tabular}{|c|c|c|c|c|c|c|c|c|}
\hline \multirow[t]{3}{*}{ Predictor } & \multicolumn{4}{|c|}{ All Patients ( $N=2222)$} & \multicolumn{4}{|c|}{ GOLD Group $D(N=5 \mid 5)$} \\
\hline & \multirow[t]{2}{*}{ OR } & \multicolumn{2}{|c|}{$95 \% \mathrm{Cl}$ of $\mathrm{OR}$} & \multirow[t]{2}{*}{ p value } & \multirow[t]{2}{*}{ OR } & \multicolumn{2}{|c|}{$95 \% \mathrm{Cl}$ of $\mathrm{OR}$} & \multirow[t]{2}{*}{ p value } \\
\hline & & Lower & Upper & & & Lower & Upper & \\
\hline \multicolumn{9}{|l|}{ Demographics } \\
\hline Age (per 10 y) & 1.182 & 1.012 & 1.380 & 0.035 & 1.386 & 1.037 & 1.852 & 0.028 \\
\hline Sex (female vs male) & 2.829 & 2.184 & 3.663 & $<0.001$ & 2.523 & 1.583 & 4.020 & $<0.001$ \\
\hline $\mathrm{BMI}\left(\mathrm{kg} / \mathrm{m}^{2}\right)$ & 0.942 & 0.917 & 0.967 & $<0.001$ & 0.942 & 0.899 & 0.986 & 0.011 \\
\hline Smoking status (active) & 0.850 & 0.625 & 1.156 & 0.300 & 0.794 & 0.410 & 1.538 & 0.494 \\
\hline \multicolumn{9}{|l|}{ Lung function } \\
\hline RV/TLC (\%) & 1.022 & 1.010 & 1.035 & $<0.001$ & 1.013 & 0.989 & 1.036 & 0.286 \\
\hline \multicolumn{9}{|l|}{ Comorbidities } \\
\hline Asthma & 1.643 & 1.232 & 2.190 & 0.001 & 1.309 & 0.782 & 2.190 & 0.306 \\
\hline Diabetes & 1.371 & 0.813 & 2.311 & 0.237 & 2.158 & 0.979 & 4.760 & 0.057 \\
\hline Arterial hypertension & 0.912 & 0.643 & 1.293 & 0.605 & $0.74 I$ & 0.409 & 1.342 & 0.323 \\
\hline Cardiac disease & 1.259 & 0.921 & 1.721 & 0.148 & 1.271 & 0.751 & 2.151 & 0.371 \\
\hline \multicolumn{9}{|l|}{ Medication } \\
\hline ICS & 1.199 & 0.905 & 1.590 & 0.206 & 2.789 & 1.340 & 5.808 & 0.006 \\
\hline OCS & 1.818 & 1.323 & 2.498 & $<0.001$ & 1.857 & 1.141 & 3.024 & 0.013 \\
\hline Cardiac medication & 1.723 & 1.173 & 2.531 & 0.006 & 1.546 & 0.784 & 3.049 & 0.208 \\
\hline $\begin{array}{l}\text { Anti-inflammatory diabetes } \\
\text { medication }\end{array}$ & 0.358 & 0.166 & 0.773 & 0.009 & 0.183 & 0.054 & 0.619 & 0.006 \\
\hline
\end{tabular}

Notes: Results for metformin were similar $(p<0.05)$, while those for any oral anti-diabetes medication did not indicate a significant relationship to this medication (se Figure I). In GOLD groups A, B, C and the pooled group ABC there were no significant relationships to any of the anti-diabetes medications.

Abbreviations: OR, odds ratio (= EXP[B]); $95 \% \mathrm{Cl}$, respective $95 \%$ confidence interval; RV/TLC, ratio of residual volume to total lung capacity determined by body plethysmography; ICS, inhaled corticosteroids; OCS, oral corticosteroids. 

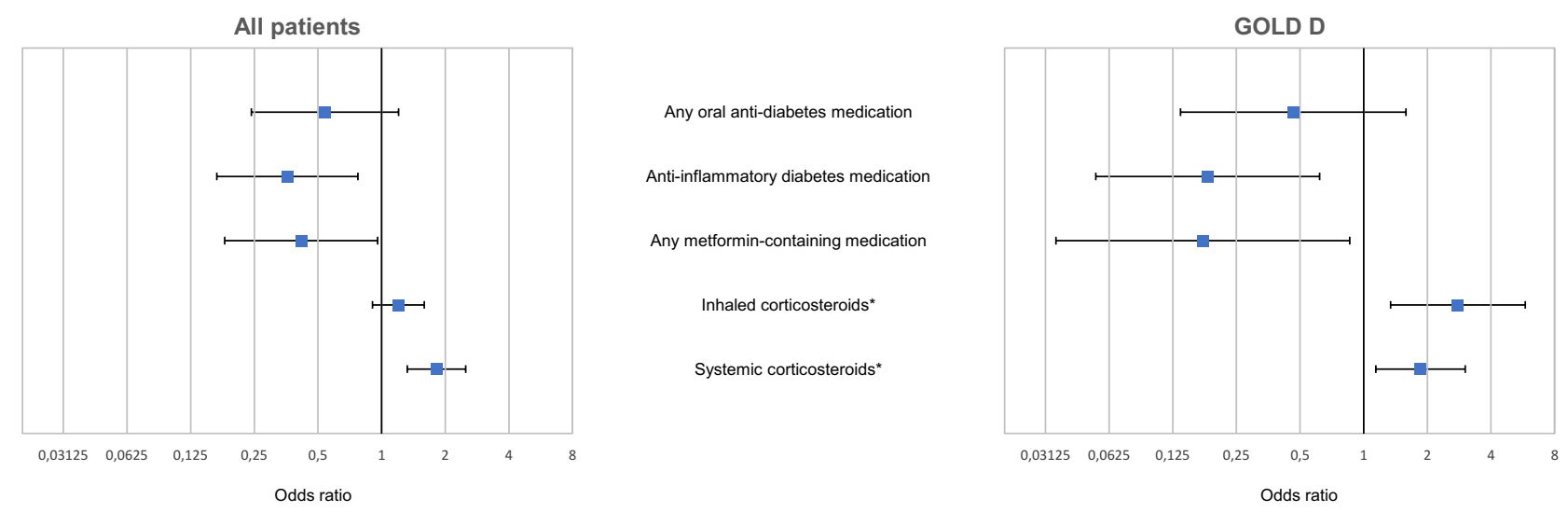

Figure I Results of logistic regression analyses in either the total population of patients or those in GOLD group D, demonstrating the significant effect of anti-inflammatory diabetes medication in both.

significant $(p=0.389)$, despite the increase in sample size. Results for GOLD group D are given in Table 3 and Figure 1.

\section{Propensity Score Matching Regarding Diabetes Medication}

In the total group, the results for ICS $(p=0.097)$ and OCS $(p=0.014)$ were in accordance with those of the conventional regression analysis, as was the case for any oral anti-diabetic medication $(\mathrm{p}=0.171)$ and for antiinflammatory anti-diabetic medication $(\mathrm{p}=0.016)$; the result for metformin only showed a tendency $(\mathrm{p}=$ 0.058). In patients in GOLD group $\mathrm{D}$, results were also consistent with the conventional regression analysis, since ICS $(p=0.022)$, OCS $(p=0.0007)$, anti-inflammatory anti-diabetic medication $(\mathrm{p}=0.004)$, and metformincontaining medication $(\mathrm{p}=0.004)$ were linked to osteoporosis, whereas any oral anti-diabetic medication was not $(\mathrm{p}=0.917)$.

\section{Discussion}

In the present cross-sectional study of patients with stable COPD, the use of anti-inflammatory compounds for the treatment of diabetes was associated with a lower prevalence of osteoporosis. This result was obtained while taking into account known osteoporosis risk factors and was robust, as it was observed with two statistical approaches. The beneficial effect of anti-diabetic medication was driven by patients from GOLD group $\mathrm{D}$, ie, patients with high symptoms and exacerbations, in whom OCS and ICS use were most prevalent, and both OCS and ICS were significantly associated with osteoporosis. Our findings suggest benefits from the treatment of diabetes in patients with COPD that extend beyond diabetes itself. This could be clinically relevant, as patients with COPD are more than twice as likely as those without COPD to have osteoporosis (OR, 2.83), with an estimated global osteoporosis prevalence of $38 \%$ in $\mathrm{COPD}^{31}$ In our study cohort, the prevalence was lower than this $(16 \%)$, probably due to the fact that our population comprised more males than females and the prevalence of osteoporosis was higher in females $(25.6 \%)$ than males $(9.9 \%)$. The development of osteoporosis is known to depend on generic risk factors including female sex, older age and low BMI, ${ }^{32-35}$ as well as COPD-related factors including smoking, ${ }^{36}$ OCS use, ${ }^{3,37}$ ICS use, ${ }^{38}$ cardiac disease $^{39}$ and diabetes. ${ }^{40} \mathrm{We}$ confirmed these associations, although, when taking into account a broad panel of risk factors, cardiac disease (20.8\% of patients) was not significantly associated with osteoporosis. It was, however, indirectly related to an increased prevalence of osteoporosis via the intake of cardiac medication including diuretics, known risk factors for osteoporosis. ${ }^{41,42}$ Although in the total study population there was no significant link between ICS use and osteoporosis, this link was clearly present in GOLD group D. It was not detectable in any of the other GOLD groups, confirming the adequacy of a stratified analysis. For this stratification, we selected GOLD groups based on the assumption that risk factors of osteoporosis including systemic inflammation would be most pronounced in patients of GOLD group D.

Systemic inflammation is not only involved in the pathophysiology of COPD, but is also relevant in diabetes and osteoporosis. ${ }^{43}$ Our observations are consistent with experimental data on the effects of anti-inflammatory antidiabetes medication, including metformin, on the risk for osteoporosis especially in the presence of corticosteroids. 
Studies demonstrated anti-inflammatory effects of antidiabetes treatment on vascular disorders, ${ }^{44}$ via inhibition of adenosine monophosphate-activated protein kinase (AMPK), production of reactive oxygen species, inhibition of interleukin (IL)-1 $\beta$ activation in macrophages, peroxisome proliferator-activated receptor (PPAR) $\gamma$, expression of pro-inflammatory cytokines and increased adiponectin levels. ${ }^{15,44,45}$ As some of these mechanisms could also be involved in the development of osteoporosis, it is reasonable to expect beneficial effects of medication with antiinflammatory action, as observed in our study. Indeed, from health insurance data there are hints that the prevalence of osteoporosis in diabetes is lower in patients receiving metformin therapy than in patients with diabetes not receiving metformin. ${ }^{46,47}$ Metformin use was also associated with a lower risk of osteoporosis regardless of the presence of diabetes or obesity. ${ }^{48}$ Indeed in a Phase 2 trial, non-diabetic patients receiving systemic glucocorticoids due to inflammatory disease were randomized to receive metformin or placebo; in the metformin arm, an improved bone metabolism was observed. ${ }^{24}$

In our study, the anti-inflammatory treatment was predominantly metformin, a drug with pleiotropic, antiinflammatory effects with the potential to prevent osteoporosis. $^{13,16,49}$ This is relevant as diabetes is linked to secondary bone loss, possibly from impaired osteoblastic function, ${ }^{50}$ and cohort studies indicated an increased risk of fracture in patients with diabetes. ${ }^{51}$ Conversely, experimental studies in animals have shown positive effects of metformin on bone loss, ${ }^{23,24}$ and treatment with oral anti-diabetic medication seems to be associated with increased density bone mineral density, in contrast to insulin, for which lower intestinal absorption and increased urinary excretion of calcium, ${ }^{16}$ as well as vitamin $\mathrm{D}$ regulation, might play a detrimental role. In cell cultures, osteogenic effects of metformin have been shown, ${ }^{7,8}$ involving AMPK, mTOR (mechanistic target of rapamycin) complexes 1 and 2 and SIRT6 (sirtuin 6), with effects on NF- $\mathrm{BB}$ (nuclear factor kappa-light-chainenhancer of activated B cells), a key pro-inflammatory transcription factor. It also increased OPG (osteoprotegerin) and decreased RANKL (receptor activator of NF$\kappa \mathrm{B}$ ligand) that is associated with osteoclastic activity and bone resorption. ${ }^{52,53}$ Thus, there are multiple pathways by which anti-inflammatory anti-diabetic medication, ${ }^{44}$ including metformin, could reduce the risk of osteoporosis. It was not the aim of the present study to identify a specific compound as protective but the results suggest that metformin played a role. These mechanisms could be especially important in patients with COPD, a large percentage of whom are treated with corticosteroids. ${ }^{18}$ Effects of corticosteroids on the development of osteoporosis are probably to be most pronounced in patients with the highest intensity of this treatment and the highest degree of systemic inflammation. From this perspective, it does not seem surprising that the beneficial effect of diabetes medication that we observed was driven by patients in GOLD group D, although this represented only about one quarter of our population.

Impaired lung function has been reported to be linked to osteoporosis; ${ }^{54}$ this was confirmed in our study, in which RV/TLC, as a marker of trapped air and hyperinflation, was the most robust lung function predictor. RV/TLC is associated with airway obstruction, ${ }^{55}$ but its predictive value dominated over that of $\mathrm{FEV}_{1}$ and FVC. Low BMI was also a risk factor for osteoporosis, although interestingly it is commonly linked to a lower, not a higher value of RV/TLC. ${ }^{56}$ These results highlight that BMI and RV/ TLC had independent predictive values. As an indicator of air trapping, RV/TLC is associated with lung emphysema, which is known to be linked to lower BMI. The fact that $\mathrm{RV} / \mathrm{TLC}$ was positively related to the prevalence of osteoporosis confirmed that lung emphysema is among the risk factors of osteoporosis. ${ }^{57}$ We additionally observed a trend for lower prevalence of diabetes among patients with osteoporosis. A potential reason for this could be the fact that COPD patients with osteoporosis had a lower BMI and thus a lower risk of developing type 2 diabetes, which was the pre-dominant type of diabetes in the cohort.

In the total group of patients, the intake of antiinflammatory anti-diabetic medication was associated with a reduction in the prevalence of osteoporosis of approximately 3-fold, while in patients of GOLD group $\mathrm{D}$ the reduction was approximately 5-fold; for metformin the reductions were approximately 2.5 -fold. These numbers indicate a marked reduction in the prevalence of osteoporosis, and should be compared with other influencing factors. The increase in prevalence associated with female sex was at least 2.5-fold, while that for OCS use was about 2-fold. In patients of GOLD group D, ICS use was linked to a close to 3-fold increase in prevalence, whereas cardiac medication was significant only in the total group, with a 1.7-fold increased osteoporosis prevalence. These numbers indicate that the benefit from antiinflammatory anti-diabetic medication was at least as great as the detrimental effect of a number of risk factors. Our 
observations suggest that future studies should carefully observe whether such tremendous effects can be substantiated.

\section{Limitations and Strengths}

Our analyses were based on cross-sectional data. Although the results are plausible from clinical and pathophysiological points of view, this does not allow us to infer causal relationships. Further, data on daily dosages, duration of treatment and adherence to medication were not collected, although from a previous analysis of COSYCONET we know that adherence to medication is very high. ${ }^{58}$ The multiple influencing factors on osteoporosis required multiple adjustments, but the results were robust in the sense that standard multiple logistic regression analysis and propensity score matching essentially yielded the same results. Finally, no measurements on bone density were available, and thus we had to rely on the patients' reports of physician-based diagnoses of osteoporosis. The same was true for other comorbidities, but those other comorbidities could be largely confirmed by analysis of diseasespecific medication. ${ }^{29}$ Still, the potential bias from a potentially sex-dependent under-diagnosis of osteoporosis has to be considered. In our study cohort, the prevalence of treatment with ICS and OCS was high as previously described. ${ }^{17,18}$ As animal data suggest a particular effectiveness against metformin-induced corticosteroid induced osteoporosis, ${ }^{13}$ it might be speculated that the beneficial effect observed by us was linked to the high level of corticosteroid treatment. We did not test whether in the GOLD group D the beneficial effect was also present in patients without corticosteroids, as the number of these patients was too small and they probably represented a highly biased sub-population. We also believe with the introduction of triple-therapy the corticosteroid-associated problems will remain in the future.

\section{Conclusion}

The present analyses indicate a beneficial effect of antiinflammatory anti-diabetic medication on the prevalence of osteoporosis in patients with COPD. This effect was especially notable in patients with frequent exacerbations and high symptoms scores, known as GOLD group D, who also had the highest exposure to ICS and OCS. The findings are consistent with experimental data regarding anti-diabetes medication, especially metformin, and highlight the pleiotropic potential of systemic medication to exert beneficial effects on other comorbidities in patients with COPD.

\section{COSYCONET Study Group}

Andreas, Stefan (Lungenfachklinik, Immenhausen); Bals, Robert (Universitätsklinikum des Saarlandes); Behr, Jürgen and Kahnert, Kathrin (Klinikum der LudwigMaximilians-Universität München); Bahmer, Thomas (Universitätsklinikum Schleswig Holstein) and Bewig, Burkhard (Städtisches Krankenhaus Kiel); Ewert, Ralf and Stubbe, Beate (Universitätsmedizin Greifswald); Ficker, Joachim H. (Klinikum Nürnberg, Paracelsus Medizinische Privatuniversität Nürnberg); Grohé, Christian (Ev. Lungenklinik Berlin); Held, Matthias (Klinikum Würzburg Mitte gGmbH, Standort Missioklinik); Behr, Jürgen and Henke, Markus (Asklepios Fachkliniken München-Gauting); Herth, Felix (Thoraxklinik Heidelberg gGmbH); Kirsten, Anne-Marie and Watz, Henrik (Pneumologisches Forschungsinstitut an der Lungenclinic Grosshansdorf GmbH); Koczulla, Rembert (Schön Klinik Berchtesgadener Land); Kronsbein, Juliane (Berufsgenossenschaftliches Universitätsklinikum Bergmannsheil, Bochum); KropfSanchen, Cornelia (Universitätsklinikum Ulm); Herzmann, Christian (Forschungszentrum Borstel); Pfeifer, Michael (Klinik Donaustauf); Randerath, Winfried J. (Wissenschaftliches Institut Bethanien e. V., Solingen); Seeger, Werner (Justus-Liebig-Universität Gießen); Studnicka, Michael (Uniklinikum Salzburg); Taube, Christian (Ruhrlandklinik gGmbH Essen); Timmermann, Hartmut (Hamburger Institut für Therapieforschung GmbH); Alter, Peter; Schmeck, Bernd and Vogelmeier, Claus (Universitätsklinikum Gießen und Marburg GmbH, Standort Marburg); Welte, Tobias (Medizinische Hochschule Hannover); Wirtz, Hubert (Universitätsklinikum Leipzig)

The study was based on data from 2741 patients recruited within the COSYCONET framework (ClinicalTrials.gov, Identifier: NCT01245933). For further information see Karch A, Vogelmeier C, Welte T, Bals R, Kauczor HU, Biederer J, Heinrich J, Schulz H, Glaser S, Holle $\mathrm{R}$ et al. The German COPD cohort COSYCONET: Aims, methods and descriptive analysis of the study population at baseline. Respir Med. 2016;114:27-37.

\section{Data Sharing Statement}

The basic data are part of the German COPD cohort COSYCONET (www.asconet.net) and available upon 
request. The website of the network provides a detailed procedure for respective applications. The data can be obtained after submission of a proposal that is evaluated by the steering committee. All results to which the manuscript refers, are documented appropriately in the text, figures or tables.

\section{Ethics Approval and Consent to Participate}

All assessments were approved by the central (Marburg (Ethikkommission FB Medizin Marburg) and local (Bad Reichenhall (Ethikkommission bayerische Landesärztekammer); Berlin (Ethikkommission Ärztekammer Berlin); Bochum (Ethikkommission Medizinische Fakultät der RUB); Borstel (Ethikkommission Universität Lübeck); Coswig (Ethikkommission TU Dresden); Donaustauf (Ethikkommission Universitätsklinikum Regensburg); Essen (Ethikkommission Medizinische Fakultät Duisburg-Essen); Gießen (Ethikkommission Fachbereich Medizin); Greifswald (Ethikkommission Universitätsmedizin Greifswald); Großhansdorf (Ethikkommission Ärztekammer Schleswig-Holstein); Hamburg (Ethikkommission Ärztekammer Hamburg); MHH Hannover/Coppenbrügge (MHH Ethikkommission); Heidelberg Thorax/Uniklinik (Ethikkommission Universität Heidelberg); Homburg (Ethikkommission Saarbrücken); Immenhausen (Ethikkommission Landesärztekammer Hessen); Kiel (Ethikkommission Christian-AlbrechtsUniversität zu Kiel); Leipzig (Ethikkommission Universität Leipzig); Löwenstein (Ethikkommission Landesärztekammer Baden-Württemberg); Mainz (Ethikkommission Landesärztekammer Rheinland-Pfalz); München LMU/ Gauting (Ethikkommission Klinikum Universität München); Nürnberg (Ethikkommission Friedrich-Alexander-Universität Erlangen Nürnberg); Rostock (Ethikkommission Universität Rostock); Berchtesgadener Land (Ethikkommission Land Salzburg); Schmallenberg (Ethikkommission Ärztekammer Westfalen-Lippe); Solingen (Ethikkommission Universität Witten-Herdecke); Ulm (Ethikkommission Universität Ulm); Würzburg (Ethikkommission Universität Würzburg)) Ethical Committees, and written informed consent was obtained from all patients.

\section{Consent for Publication}

Within the ethical approval of COSYCONET, the participants of the study gave their consent to publish the data collected without reference to their person.

\section{Acknowledgments}

We are grateful to all COSYCONET study centers, especially to all study nurses, for their excellent and enduring work in data collection, as well as to all patients who were willing to participate in this study. COSYCONET is supported by the German Federal Ministry of Education and Research (BMBF) Competence Network Asthma and COPD (ASCONET) and performed in collaboration with the German Center for Lung Research (DZL). The project is funded by the BMBF with grant number 01 GI 0881, and is supported by unrestricted grants from AstraZeneca $\mathrm{GmbH}$, Bayer Schering Pharma AG, Boehringer Ingelheim Pharma $\mathrm{GmbH} \& \mathrm{Co}$. KG, Chiesi $\mathrm{GmbH}$, GlaxoSmithKline, Grifols Deutschland GmbH, MSD Sharp \& Dohme GmbH, Mundipharma GmbH, Novartis Deutschland GmbH, Pfizer Pharma GmbH, Takeda Pharma Vertrieb $\mathrm{GmbH} \& \mathrm{Co}$. KG, Teva $\mathrm{GmbH}$ for patient investigations and laboratory measurements. The funding body had no involvement in the design of the study, or the collection, analysis or interpretation of the data. We thank Christina Kellerer, TU Munich, for her help in propensity score analysis. We thank David Young for helpful comments on the manuscript.

\section{Author Contributions}

All authors made a significant contribution to the work reported, whether that is in the conception, study design, execution, acquisition of data, analysis and interpretation, or in all these areas; took part in drafting, revising or critically reviewing the article; gave final approval of the version to be published; have agreed on the journal to which the article has been submitted; and agree to be accountable for all aspects of the work.

\section{Disclosure}

Professor Joachim H Ficker reports personal fees from Boehringer-Ingelheim, AstraZeneca, and GSK; personal fees, non-financial support from CSL Behring and Novartis, outside the submitted work. Professor Jürgen Behr reports personal fees from Boehringer-Ingelheim, AstraZeneca, and Novartis, outside the submitted work. Professor Robert Bals reports COSYCONET is supported by the German Centre for Lung Research (DZL), grant number 82DZLI05A2 (COSYCONET), the BMBF, grant number 01GI0881 and also received unrestricted grants from AstraZeneca $\mathrm{GmbH}$, Boehringer Ingelheim Pharma $\mathrm{GmbH} \&$ Co. KG, GlaxoSmithKline GmbH\&Co. KG, 
Grifols Deutschland GmbH, and Novartis Deutschland $\mathrm{GmbH}$. Professor Bals also reports grants, and/or personal fees from AstraZeneca, Boehringer Ingelheim, GlaxoSmithKline, Grifols, Novartis, CSL Behring, German Federal Ministry of Education and Research (BMBF) Competence Network Asthma and COPD (ASCONET), Sander Stiftung, Schwiete Stiftung, Krebshilfe, and Mukoviszidose eV, outside the submitted work. Professor Claus F Vogelmeier reports personal fees, and/or grants from Aerogen, AstraZeneca, Boehringer Ingelheim, CSL Behring, Chiesi, GlaxoSmithKline, Grifols, Menarini, Novartis, Nuvaira, and MedUpdate, outside the submitted work. The authors declare that they have no other competing interests.

\section{References}

1. Franco CB, Paz-Filho G, Gomes PE, et al. Chronic obstructive pulmonary disease is associated with osteoporosis and low levels of vitamin D. Osteoporos Int. 2009;20(11):1881-1887. doi:10.1007/ s00198-009-0890-5

2. Vanfleteren LE, Spruit MA, Groenen M, et al. Clusters of comorbidities based on validated objective measurements and systemic inflammation in patients with chronic obstructive pulmonary disease. $\mathrm{Am}$ $J$ Respir Crit Care Med. 2013;187(7):728-735. doi:10.1164/ rccm.201209-1665OC

3. Sarkar M, Bhardwaj R, Madabhavi I, Khatana J. Osteoporosis in chronic obstructive pulmonary disease. Clin Med Insights Circ Respir Pulm Med. 2015;9:5-21. doi:10.4137/CCRPM.S22803

4. Karch A, Vogelmeier C, Welte T, et al. The German COPD cohort COSYCONET: aims, methods and descriptive analysis of the study population at baseline. Respir Med. 2016;114:27-37. doi:10.1016/j. rmed.2016.03.008

5. Okazaki R, Watanabe R, Inoue D. Osteoporosis associated with chronic obstructive pulmonary disease. J Bone Metab. 2016;23 (3):111-120. doi:10.11005/jbm.2016.23.3.111

6. Nayyar N, Sood RG, Sarkar M, Tomar A, Thakur V, Bhoil R. Prevalence of osteoporosis and osteopenia in stable patients of chronic obstructive pulmonary disease in Sub-Himalayan region of Himachal Pradesh, India. J Family Med Prim Care. 2017;6 (3):595-599. doi:10.4103/2249-4863.222013

7. Liao KM, Chiu KL, Chen CY. Prescription patterns in patients with chronic obstructive pulmonary disease and osteoporosis. Int J Chron Obstruct Pulmon Dis. 2021;16:761-769. doi:10.2147/COPD. S289799

8. Inoue D, Watanabe R, Okazaki R. COPD and osteoporosis: links, risks, and treatment challenges. Int J Chron Obstruct Pulmon Dis. 2016;11:637-648. doi:10.2147/COPD.S79638

9. Bikov A, Horvath A, Tomisa G, Bartfai L, Bartfai Z. Changes in the burden of comorbidities in patients with COPD and asthma-COPD overlap according to the GOLD 2017 recommendations. Lung. 2018;196(5):591-599. doi:10.1007/s00408-018-0141-7

10. Liang B, Feng Y. The association of low bone mineral density with systemic inflammation in clinically stable COPD. Endocrine. 2012;42(1):190-195. doi:10.1007/s12020-011-9583-x

11. Kahnert K, Lucke T, Biertz F, et al. Transfer factor for carbon monoxide in patients with COPD and diabetes: results from the German COSYCONET cohort. Respir Res. 2017;18(1):14. doi:10.1186/s12931-016-0499-0
12. Tsalamandris S, Antonopoulos AS, Oikonomou E, et al. The role of inflammation in diabetes: current concepts and future perspectives. Eur Cardiol. 2019;14(1):50-59. doi:10.15420/ecr.2018.33.1

13. Aung M, Amin S, Gulraiz A, Gandhi FR, Pena Escobar JA, Malik BH. The future of metformin in the prevention of diabetes-related osteoporosis. Cureus. 2020;12(9):e10412.

14. Kalaitzidis RG, Sarafidis PA, Bakris GL. Effects of thiazolidinediones beyond glycaemic control. Curr Pharm Des. 2009;15 (5):529-536. doi:10.2174/138161209787315693

15. Scheen AJ, Esser N, Paquot N. Antidiabetic agents: potential anti-inflammatory activity beyond glucose control. Diabetes Metab. 2015;41(3):183-194. doi:10.1016/j.diabet.2015.02.003

16. Cortet B, Lucas S, Legroux-Gerot I, Penel G, Chauveau C, Paccou J. Bone disorders associated with diabetes mellitus and its treatments. Joint Bone Spine. 2019;86(3):315-320. doi:10.1016/j. jbspin.2018.08.002

17. Lutter JI, Jorres RA, Trudzinski FC, et al. Treatment of COPD groups GOLD A and B with inhaled corticosteroids in the COSYCONET cohort - determinants and consequences. Int J Chron Obstruct Pulmon Dis. 2021;16:987-998. doi:10.2147/COPD.S304532

18. Graf J, Jorres RA, Lucke T, Nowak D, Vogelmeier CF, Ficker JH. Medical treatment of COPD. Dtsch Arztebl Int. 2018;155 (37):599-605.

19. Compston J. Glucocorticoid-induced osteoporosis: an update. Endocrine. 2018;61(1):7-16. doi:10.1007/s12020-018-1588-2

20. Kumarathas I, Harslof T, Andersen CU, et al. The risk of osteoporosis in patients with asthma. Eur Clin Respir J. 2020;7(1):1763612. doi:10.1080/20018525.2020.1763612

21. Cortizo AM, Sedlinsky C, McCarthy AD, Blanco A, Schurman L. Osteogenic actions of the anti-diabetic drug metformin on osteoblasts in culture. Eur J Pharmacol. 2006;536(1-2):38-46. doi:10.1016/j. ejphar.2006.02.030

22. Kanazawa I, Yamaguchi T, Yano S, Yamauchi M, Sugimoto T. Metformin enhances the differentiation and mineralization of osteoblastic MC3T3-E1 cells via AMP kinase activation as well as eNOS and BMP-2 expression. Biochem Biophys Res Commun. 2008;375 (3):414-419. doi:10.1016/j.bbrc.2008.08.034

23. Gao Y, Li Y, Xue J, Jia Y, Hu J. Effect of the anti-diabetic drug metformin on bone mass in ovariectomized rats. Eur J Pharmacol. 2010;635(1-3):231-236. doi:10.1016/j.ejphar.2010.02.051

24. Stunes AK, Erben RG, Schuler C, et al. Skeletal effects of plyometric exercise and metformin in ovariectomized rats. Bone. 2020;132:115193. doi:10.1016/j.bone.2019.115193

25. Zhao J, Li Y, Zhang H, et al. Preventative effects of metformin on glucocorticoid-induced osteoporosis in rats. J Bone Miner Metab. 2019;37(5):805-814. doi:10.1007/s00774-019-00989-y

26. Vogelmeier CF, Criner GJ, Martinez FJ, et al. Global strategy for the diagnosis, management, and prevention of chronic obstructive lung disease 2017 report: GOLD executive summary. Eur Respir J. 2017;49(3):1700214. doi:10.1183/13993003.00214-2017

27. Quanjer PH, Stanojevic S, Cole TJ, et al. Multi-ethnic reference values for spirometry for the 3-95-yr age range: the global lung function 2012 equations. Eur Respir J. 2012;40(6):1324-1343. doi:10.1183/09031936.00080312

28. Singh D, Agusti A, Anzueto A, et al. Global strategy for the diagnosis, management, and prevention of chronic obstructive lung disease: the GOLD science committee report 2019. Eur Respir J. 2019;53(5):1900164. doi:10.1183/13993003.00164-2019

29. Lucke T, Herrera R, Wacker M, et al. Systematic analysis of self-reported comorbidities in large cohort studies - a novel stepwise approach by evaluation of medication. PLoS One. 2016;11(10): e0163408. doi:10.1371/journal.pone.0163408

30. Kellerer C, Kahnert K, Trudzinski FC, et al. COPD maintenance medication is linked to left atrial size: results from the COSYCONET cohort. Respir Med. 2021;185:106461. 
31. Chen YW, Ramsook AH, Coxson HO, Bon J, Reid WD. Prevalence and risk factors for osteoporosis in individuals with COPD: a systematic review and meta-analysis. Chest. 2019;156 (6):1092-1110. doi:10.1016/j.chest.2019.06.036

32. De Laet C, Kanis JA, Oden A, et al. Body mass index as a predictor of fracture risk: a meta-analysis. Osteoporos Int. 2005;16 (11):1330-1338. doi:10.1007/s00198-005-1863-y

33. Xiang BY, Huang W, Zhou GQ, Hu N, Chen H, Chen C. Body mass index and the risk of low bone mass-related fractures in women compared with men: a PRISMA-compliant meta-analysis of prospective cohort studies. Medicine. 2017;96(12):e5290. doi:10.1097/ MD.0000000000005290

34. Hariri AF, Almatrafi MN, Zamka AB, et al. Relationship between body mass index and T-scores of bone mineral density in the hip and spine regions among older adults with diabetes: a retrospective review. J Obes. 2019;2019:9827403. doi:10.1155/2019/9827403

35. Xuan R, Song Y, Baker JS, Gu Y. The evaluation of bone mineral density based on age and anthropometric parameters in Southeast Chinese adults: a cross-sectional study. Med Sci Monit. 2020;26: e923603. doi:10.12659/MSM.923603

36. Bon J, Nouraie SM, Smith KJ, et al. Lung-specific risk factors associated with incident hip fracture in current and former smokers. J Bone Miner Res. 2020;35(10):1952-1961. doi:10.1002/jbmr.4103

37. Lu PC, Yang YH, Guo SE, Yang TM. Factors associated with osteoporosis in patients with chronic obstructive pulmonary disease-a nationwide retrospective study. Osteoporos Int. 2017;28 (1):359-367. doi:10.1007/s00198-016-3732-2

38. Chiu KL, Lee CC, Chen CY. Evaluating the association of osteoporosis with inhaled corticosteroid use in chronic obstructive pulmonary disease in Taiwan. Sci Rep. 2021;11(1):724. doi:10.1038/ s41598-020-80815-y

39. den Uyl D, Nurmohamed MT, van Tuyl LH, Raterman HG, Lems WF. (Sub)clinical cardiovascular disease is associated with increased bone loss and fracture risk; a systematic review of the association between cardiovascular disease and osteoporosis. Arthritis Res Ther. 2011;13(1):R5. doi:10.1186/ar3224

40. Leidig-Bruckner G, Grobholz S, Bruckner T, Scheidt-Nave C, Nawroth P, Schneider JG. Prevalence and determinants of osteoporosis in patients with type 1 and type 2 diabetes mellitus. BMC Endocr Disord. 2014;14:33. doi:10.1186/1472-6823-14-33

41. Kelsey JL. Risk factors for osteoporosis and associated fractures. Public Health Rep. 1989;104 Suppl(Suppl):14-20.

42. Rejnmark L, Mosekilde L, Andreasen F. [Diuretics and osteoporosis]. Nord Med. 1998;113(2):53-59. Danish.

43. Miller J, Edwards LD, Agusti A, et al. Comorbidity, systemic inflammation and outcomes in the ECLIPSE cohort. Respir Med. 2013;107 (9):1376-1384. doi:10.1016/j.rmed.2013.05.001

44. Pollack RM, Donath MY, LeRoith D, Leibowitz G. Antiinflammatory agents in the treatment of diabetes and its vascular complications. Diabetes Care. 2016;39(Suppl 2):S244-S252. doi: $10.2337 / \mathrm{dcS} 15-3015$
45. Yaribeygi H, Atkin SL, Pirro M, Sahebkar A. A review of the anti-inflammatory properties of antidiabetic agents providing protective effects against vascular complications in diabetes. $J$ Cell Physiol. 2019;234(6):8286-8294. doi:10.1002/jcp.27699

46. Lu CH, Chung CH, Kuo FC, et al. Metformin attenuates osteoporosis in diabetic patients with carcinoma in situ: a nationwide, retrospective, matched-cohort study in Taiwan. J Clin Med. 2020;9(9):2839. doi: $10.3390 /$ jem 9092839

47. Tseng $\mathrm{CH}$. Metformin use is associated with a lower risk of osteoporosis/ vertebral fracture in Taiwanese patients with type 2 diabetes mellitus. Eur J Endocrinol. 2021;184(2):299-310. doi:10.1530/EJE-20-0507

48. Blumel JE, Arteaga E, Aedo S, et al. Metformin use is associated with a lower risk of osteoporosis in adult women independent of type 2 diabetes mellitus and obesity. REDLINC IX study. Gynecol Endocrinol. 2020;36(5):421-425. doi:10.1080/09513590.2020.1718092

49. McCarthy AD, Cortizo AM, Sedlinsky C. Metformin revisited: does this regulator of AMP-activated protein kinase secondarily affect bone metabolism and prevent diabetic osteopathy. World J Diabetes. 2016;7(6):122-133. doi:10.4239/wjd.v7.i6.122

50. Chau DL, Edelman SV, Chandran M. Osteoporosis and diabetes. Curr Diab Rep. 2003;3(1):37-42. doi:10.1007/s11892-003-0051-8

51. Carnevale V, Romagnoli E, D'Erasmo E. Skeletal involvement in patients with diabetes mellitus. Diabetes Metab Res Rev. 2004;20 (3):196-204. doi:10.1002/dmrr.449

52. Mai QG, Zhang ZM, Xu S, et al. Metformin stimulates osteoprotegerin and reduces RANKL expression in osteoblasts and ovariectomized rats. J Cell Biochem. 2011;112(10):2902-2909. doi:10.1002/ jcb. 23206

53. Mu W, Wang Z, Ma C, et al. Metformin promotes the proliferation and differentiation of murine preosteoblast by regulating the expression of sirt6 and oct4. Pharmacol Res. 2018;129:462-474. doi:10.1016/j.phrs.2017.11.020

54. Leech JA, Dulberg C, Kellie S, Pattee L, Gay J. Relationship of lung function to severity of osteoporosis in women. Am Rev Respir Dis. 1990;141(1):68-71. doi:10.1164/ajrccm/141.1.68

55. Alter P, Orszag J, Kellerer C, et al. Prediction of air trapping or pulmonary hyperinflation by forced spirometry in COPD patients: results from COSYCONET. ERJ Open Res. 2020;6(3):00092-2020. doi:10.1183/23120541.00092-2020

56. Park HJ, Cho JH, Kim HJ, Park JY, Lee HS, Byun MK. The effect of low body mass index on the development of chronic obstructive pulmonary disease and mortality. $J$ Intern Med. 2019;286 (5):573-582. doi:10.1111/joim.12949

57. Ohara T, Hirai T, Muro S, et al. Relationship between pulmonary emphysema and osteoporosis assessed by CT in patients with COPD. Chest. 2008;134(6):1244-1249. doi:10.1378/chest.07-3054

58. Konigsdorfer N, Jorres RA, Sohler S, et al. Adherence to respiratory and nonrespiratory medication in patients with COPD: results of the German COSYCONET cohort. Patient Prefer Adherence. 2019;13:1711-1721.

\section{Publish your work in this journal}

The International Journal of COPD is an international, peer-reviewed journal of therapeutics and pharmacology focusing on concise rapid reporting of clinical studies and reviews in COPD. Special focus is given to the pathophysiological processes underlying the disease, intervention programs, patient focused education, and self management protocols. This journal is indexed on PubMed Central, MedLine and CAS. The manuscript management system is completely online and includes a very quick and fair peer-review system, which is all easy to use. Visit http://www.dovepress.com/testimonials.php to read real quotes from published authors. 